\title{
Etude ethnopharmacologique des plantes utilisées pour faciliter l'accouchement, en pays Abbey et Krobou, au Sud de la Côte-d'Ivoire
}

\author{
Koffi N’GUESSAN *, Noël Guédé ZIRIHI et N'Takpé Kama Maxime BORAUD \\ Université de Cocody-Abidjan, U.F.R. Biosciences, Laboratoire de Botanique. 22 BP 582 Abidjan 22, Côte- \\ d'Ivoire. \\ *Auteur correspondant, E-mail: nguessankoffifr@yahoo.fr ; Tel: (225) 07873013
}

\section{RESUME}

Les populations rurales africaines, pour éviter la tragédie de l'accouchement, se tournent vers les plantes, du fait de l'absence des centres sanitaires, de leur éloignement ou du manque de moyens financiers. Dans le but de rechercher des thérapeutiques faciles d'accès et moins onéreuses, nous avons entrepris une étude ethnopharmacologique à Agboville (Côte-d'Ivoire). Elle a permis de visiter 14 villages et de rencontrer, lors des entretiens semi structurés, 80 tradithérapeutes. Cette étude montre que les Abbey et les Krobou emploient 34 espèces de plantes, pour faciliter les accouchements. Les recettes médicamenteuses sont, en majeure partie, monospécifiques $(82,60 \%)$. Les drogues (écorces de racine, écorce de tige, feuilles, fruits, graines, inflorescences, racines et tubercule) servent à mettre au point divers remèdes, par décoction, expression, macération, pétrissage, pilage et ramollissement. Les médicaments sont administrés par boisson (45,23\%), instillations buccales $(11,90 \%)$ et purge $(42,85 \%)$. Les guérisseurs n'utilisent que les ressources naturelles (plantes, animaux et minéraux). Dans certains cas, le traitement est accompagné d'incantations. Les effets induits par les plantes sont le fait de divers groupes chimiques : acide ascorbique, adansonine, alcaloïdes indoliques, flavonoïdes, saponosides triterpéniques et vitamine $\mathrm{B}_{1}$.

(C) 2010 International Formulae Group. All rights reserved.

Mots clés : Agboville, Ethnopharmacologie, Incantation, Tradithérapeute.

\section{INTRODUCTION}

Chez les Abbey et Krobou, deux ethnies du Département d'Agboville (Côted'Ivoire), le sentiment légitime de tout couple est d'avoir au moins un enfant. Malheureusement, chaque année, dans le monde, ce sont environ 600000 femmes qui meurent des suites d'une grossesse ou d'un accouchement. La plupart de ces décès sont à déplorer en Afrique où 1 femme sur 21 trouve la mort, au cours de la grossesse ou de l'accouchement (N'guessan, 2000). Selon un rapport de l'Association Ivoirienne du Bien- être Familial, en milieu rural, $70 \%$ des femmes accouchent seules, à la maison, sinon, elles le font, assistées d'une accoucheuse traditionnelle ou d'un membre de la famille, personne généralement peu qualifiée (Anonyme, 1994). Dans ces sociétés Abbey et Krobou, les accoucheuses traditionnelles ou matrones administrent à la parturiente des préparations médicamenteuses faites souvent de drogues végétales auxquelles on associe, parfois, des organes d'animaux et des substances minérales. On pense aussi que par les incantations, il est possible d'appeler la 
bénédiction sur le médicament (N'guessan et al., 2009a). Le recours aux drogues végétales, pour faciliter l'accouchement, a été rapporté par des études menées dans diverses localités de la Côte-d'Ivoire (Kamanzi, 2002 ; Koné, 2005 ; Ouattara, 2006 ; Zirihi, 2006). Dans bon nombre de pays en voie d'émergence, particulièrement en Afrique, divers travaux ont indiqué que les drogues végétales sont employées, par des populations rurales, pour éviter la tragédie de l'accouchement (BinimbiMassengo et al., 2007; Bantsiélé et al., 2007 ; Folliard, 2008). L'absence ou l'éloignement des centres sanitaires, le manque de moyens financiers, peuvent expliquer pourquoi, au risque de leur vie, les femmes préfèrent accoucher chez elles, non assistées par un professionnel de la santé. Dans cette étude, nous faisons découvrir les plantes que l'on emploie dans ce milieu traditionnel, pour faciliter les accouchements. En accord avec la littérature, nous indiquons les bases pharmacologiques et phytochimiques qui permettent d'expliquer le bien fondé des utilisations thérapeutiques traditionnelles des plantes étudiées.

\section{MATÉRIEL ET MÉTHODES Site de l'étude}

Nos investigations ethnopharmacologiques ont été menées dans le Département d'Agboville (Figure 1). Situé à environ $80 \mathrm{~km}$ d'Abidjan, Agboville fait partie du Sud forestier de la Côte-d'Ivoire, du domaine guinéen à secteur mésophile caractérisé par la forêt dense humide semi-décidue, actuellement dégradée (N'Guessan, 2008). La pluviométrie moyenne annuelle est de l'ordre de $1400 \mathrm{~mm}$ d'eau. Juin est le mois le plus pluvieux (270 mm de pluie) et janvier, le mois le plus sec (11 mm de pluie). Le climat, de type guinéen, se caractérise par une saison sèche de décembre à février et une longue saison des pluies de mars à novembre. Le Département d'Agboville compte 220.050 habitants (Anonyme, 1999). A côté des autochtones que sont les Abbey et Krobou, deux ethnies du groupe Akan, dans le grand groupe Kwa (Sournia et Arnaud, 1978), on note une communauté d'allochtones, en provenance de toutes les régions de la Côted'Ivoire ainsi que des allogènes, originaires de la sous région Ouest africaine.

\section{Collecte du matériel végétal}

Une prospection a été effectuée dans le Département afin de rechercher, dans divers milieux écologiques, les espèces de plantes citées par les tradithérapeutes et de prélever, grâce à un matériel classique, sur des spécimens accessibles, des échantillons, pour leur mise en herbier:

Abrus precatorius (Fabaceae): AboudéMandéké, 23 mai 1990, N'guessan Koffi 165

Adansonia digitata (Bombacaceae): Agboville (Côte-d'Ivoire), 25 août 1999, N'guessan Koffi 322

Adenia lobata (Passifloraceae): AboudéMandéké, 14 février 1990, N’guessan Koffi 74

Ageratum conyzoides (Asteraceae): OressKrobou, 11 mai 1990, N'guessan Koffi 150

Alternanthera pungens (Amaranthaceae): Aboudé-Mandéké, 11 juillet 1990, N'guessan Koffi 247

Antiaris toxicaria var. africana (Moraceae) : Aboudé-Mandéké, 7 juin 1989, N'guessan Koffi 26

Antidesma laciniatum (Euphorbiaceae): Aboudé-Mandéké, 28 avril 1990, N'guessan Koffi 135

Arachis hypogaea (Fabaceae): AboudéKouassikro, 28 mai 1990, N'guessan Koffi 183

Blighia welwitschii (Sapindaceae): AboudéMandéké, 7 juillet 1989, N'guessan Koffi 45

Boerhavia diffusa (Nyctaginaceae) : Moutcho (Côte-d'Ivoire), 18 février 2000, N'guessan Koffi 339

Buchholzia coriacea (Capparidaceae): Offoumpo (Côte-d'Ivoire), 19 août 1999, N'guessan Koffi 316

Caesalpinia bonduc (Caesalpiniaceae): Aboudé-Mandéké, $1^{\text {er }}$ mai 1990, N'guessan Koffi 144

Carapa procera (Meliaceae): AboudéMandéké, 21 juin 1989, N'guessan Koffi 31 
Carpolobia lutea (Polygalaceae) Abidjan, Jard. Bot., 2 septembre 1999, N'guessan Koffi 327

Costus afer (Zingiberaceae): AboudéMandéké, 3 mars 1990, N'guessan Koffi 90

Ehretia cymosa (Boraginaceae): AboudéMandéké, 27 mai 1990, N'guessan Koffi 181

Gouania longipetala (Rhamnaceae) : AboudéMandéké, 7 juin 1989, N'guessan Koffi 21

Jatropha curcas (Euphorbiaceae): AboudéKouassikro, 5 mars 1990, N'guessan Koffi 94

Kalanchoe crenata (Crassulaceae) : AboudéKouassikro, 18 avril 1990, N'guessan Koffi 124

Lecaniodiscus cupanioides (Sapindaceae): Aboudé-Mandéké, 7 juin 1989, N'guessan Koffi 22

Myrianthus arboreus (Cecropiaceae): Aboudé-Mandéké, 3 mars 1990, N'guessan Koffi 87

Myrianthus libericus (Cecropiaceae): Aboudé-Mandéké, 3 mars 1990, N'guessan Koffi 91

Newbouldia laevis (Bignoniaceae) : AboudéMandéké, 21 juin 1989, N'guessan Koffi 29

Paullinia pinnata (Sapindaceae): AboudéMandéké, 14 avril 1990, N'guessan Koffi 114

Persea americana (Lauraceae): AboudéMandéké, 16 septembre 1999, N'guessan Koffi 337

Pterocarpus santalinoides (Fabaceae): Aboudé-Mandéké, 7 juillet 1989, N'guessan Koffi 44

Ricinodendron heudelotii (Euphorbiaceae) : Aboudé-Mandéké, 23 mai 1990, N'guessan Koffi 164

Solenostemon monostachyus (Lamiaceae) : Aboudé-Kouassikro, 21 juin 1990, N'guessan Koffi 202

Spondias mombin (Anacardiaceae) : AboudéMandéké, 19 juillet 1989, N'guessan Koffi 69

Spondianthus preussii (Euphorbiaceae): Aboudé-Mandéké, 14 février 1990, N'guessan Koffi 76

Sterculia tragacantha (Sterculiaceae) : Aboudé-Mandéké, 6 juin 1989, N'guessan Koffi 9
Strombosia pustulata var. lucida (Olacaceae) : Aboudé-Mandéké, 7 juin 1989, N'guessan Koffi 24

Treculia africana var. africana (Moraceae) : Aboudé-Mandéké, 19 avril 1990, N'guessan Koffi 125

Uapaca esculenta (Euphorbiaceae) : AboudéMandéké, 7 juin 1989, N'guessan Koffi 17.

\section{Méthodologie d'enquête ethnopharmacologique}

L'enquête sur les plantes facilitant l'accouchement a été réalisée parmi les autochtones de 14 villages du Département d'Agboville. Comme approche utilisée, nous avons rendu visite aux guérisseurs (hommes et femmes). Sur la base d'une fiche d'enquête, un questionnaire leur a été proposé. Les renseignements ont porté sur les plantes facilitant l'accouchement, les différents organes employés comme drogues, les méthodes de récolte, les techniques de préparation et d'administration des remèdes. A partir des informations recueillies au près des tradithérapeutes, nous avons attribué un indice d'efficacité à chaque plante, en fonction de la rapidité avec laquelle elle provoque l'accouchement, après son administration à la parturiente.

\section{Collecte des données botaniques et phytochimiques}

$\mathrm{Au}$ laboratoire, pour la nomenclature et les descriptions botaniques, nous nous sommes servis des échantillons récoltés, des spécimens de l'herbier du Centre National de Floristique, des flores et des ouvrages divers. La vérification des noms de taxons a été faite par le Professeur Aké-Assi Laurent, Systématicien Botaniste. Des données phytochimiques de la littérature ont permis de comprendre le bien fondé des utilisations thérapeutiques traditionnelles.

\section{Analyse statistique des données de l'enquête}

A partir des informations relatives au temps d'action des différents remèdes, nous 
avons affecté un indice d'efficacité à chaque espèce de plante, de la façon suivante :

-dans l'intervalle [ 1 à $10 \mathrm{~min}$ ], l'indice d'efficacité est estimé à 91-100

-dans l'intervalle [11 à $20 \mathrm{~min}]$, l'indice d'efficacité est estimé à 81-90

-dans l'intervalle [21 à $30 \mathrm{~min}$ ], l'indice d'efficacité est estimé à 71-80

-dans l'intervalle [31 à $40 \mathrm{~min}$ ], l'indice d'efficacité est estimé à 61-70

-dans l'intervalle [41 à $50 \mathrm{~min}$ ], l'indice d'efficacité est estimé à 51-60

-dans l'intervalle [51 à $60 \mathrm{~min}$ ], l'indice d'efficacité est estimé à 41-50

-dans l'intervalle [61 à $70 \mathrm{~min}$ ], l'indice d'efficacité est estimé à 31-40.

-dans l'intervalle [71 à $80 \mathrm{~min}]$, l'indice d'efficacité est estimé à 21-30.

Les plantes, en fonction de leur indice d'efficacité, ont été soumises à une classification hiérarchique, à l'aide du logiciel SPPSS 12.0, utilisant la méthode Ward.

\section{RESULTATS}

\section{Caractéristiques botaniques des plantes étudiées} Les

investigations ethnopharmacologiques que nous avons menées en pays Abbey et Krobou, dans le Département d'Agboville (Côte-d'Ivoire), ont permis d'inventorier 34 espèces de plantes facilitant les accouchements (Tableau 1). Ces espèces de plantes appartiennent à 33 genres et 24 familles réparties en 2 classes (Monocotylédones et Dicotylédones), 01 sousembranchement (Angiospermes) et 01 embranchement (Spermaphytes). Les Spermaphytes (34), tous des Angiospermes, sont représentés par une seule Monocotylédone (Costus afer, Zingiberaceae) et 33 Dicotylédones qui constituent $97,05 \%$ des espèces recensées. Les Euphorbiaceae, avec 05 espèces $(15,15 \%)$, constituent la famille la mieux représentée. Les Fabaceae viennent en deuxième position, avec 03 espèces. Du point de vue de leur statut, on dénombre 16 plantes sauvages et 18 plantes de milieux anthropiques qui comprennent 04 plantes cultivées, 01 plante pluviale, 04 plantes rudérales, 02 plantes de jachère et 07 plantes de forêt secondaire. Comme Types Morphologiques, nous notons des arbres (15 espèces soit 44,11\%), des arbustes (07 espèce soit $20,58 \%$ ), des herbes (07 espèces soit $20,58 \%)$ et des lianes (05 espèces soit $14,70 \%)$. Les arbres sont donc majoritairement représentés (Figure 2).

\section{Caractéristiques ethnopharmacologiques des plantes étudiées \\ $\mathrm{Au}$ cours de cette étude} ethnopharmacologique, nous avons rencontré 80 tradithérapeutes $(30$ hommes et 50 femmes), originaires de 14 villages du Département d'Agboville. Le plus vieil homme est âgé de 70 ans environ; la plus jeune femme est âgée de 30 ans. C'est à Aboudé-Mandéké que nous avons rencontré le plus grand nombre de gynécologues (12, soit $15 \%)$.

Les drogues employées sont diverses: écorces de racine, écorce de tige, feuilles, fruits, graines, inflorescence, racines, tige et tubercule (Figure 3). Les tiges et leurs écorces $(45,92 \%)$ sont majoritairement utilisées. On fait des prélèvements de ces drogues grâce à la houe, à la main, avec des cueilloirs spéciaux, grâce à l'ébranchoir. L'écorçage s'effectue grâce à des machettes pour les tiges et les racines des spécimens ligneux ; on procède par ébranchage, grâce à des cueilloirs spéciaux (ébranchoirs), pour des spécimens assez hauts. Les recettes médicamenteuses sont préparées à partir de drogues végétales, de ressources animales et minérales. Sur le plan opothérapique, on utilise des organes d'animaux comme le blanc d'œuf. Dans le domaine de la minéralothérapie, on emploie, par exemple, le kaolin. On distingue divers modes de préparation : décoction, expression, macération, pétrissage, pilage et ramollissement. Le pétrissage (45\%) est le mode de préparation le plus sollicité. On note 23 remèdes dont $19 \quad(82,60 \%)$ sont monospécifiques. Les formes médicamenteuses sont diverses : décoctés, jus extraits des écorces de tige, de racine ou de feuilles, macérés, mixtures aqueuses, mixtures 
au vin de palme et mixtures au jus de citron. Ces remèdes sont administrés en boisson $(45,23 \%)$, en instillations buccales $(11,90 \%)$ et en purge $(42,85 \%)$.

Les indices d'efficacité, déterminés à partir de la rapidité avec laquelle on observe l'effet des remèdes, après leur administration, sont consignés dans le Tableau 1. L'analyse descriptive est représentée sous forme de dendrogramme (Figure 4).

\section{Données phytochimiques et pharmacolo- giques}

Des données phytochimiques et pharmacologiques de la littérature ont permis de comprendre le bien fondé des utilisations thérapeutiques traditionnelles.

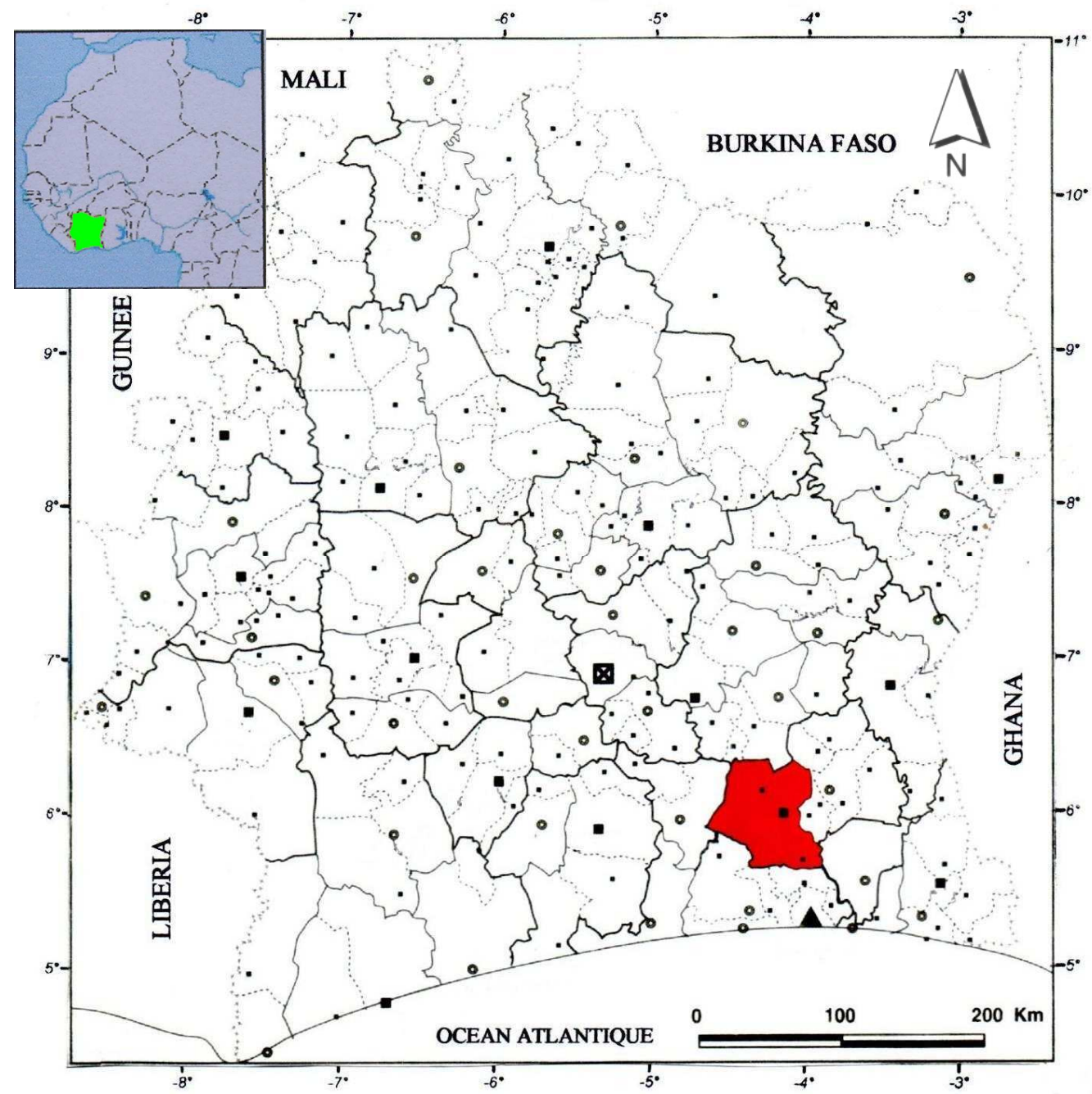

Situation géographique de la Côte-d'Ivoire en Afrique de l'Ouest

Situation géographique du Département d'Agboville en Côte-d'Ivoire

Figure 1 : Site de l'étude (CEDA, 2001, modifiée par N'guessan). 


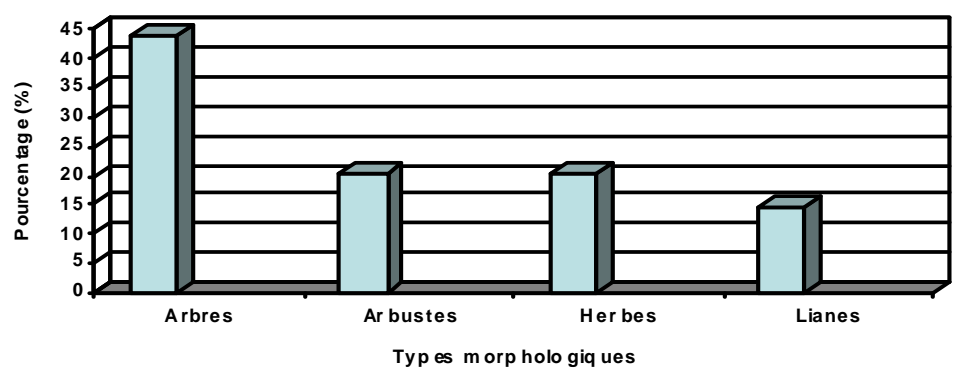

Figure 2 : Histogramme de la répartition des Types Morphologiques.

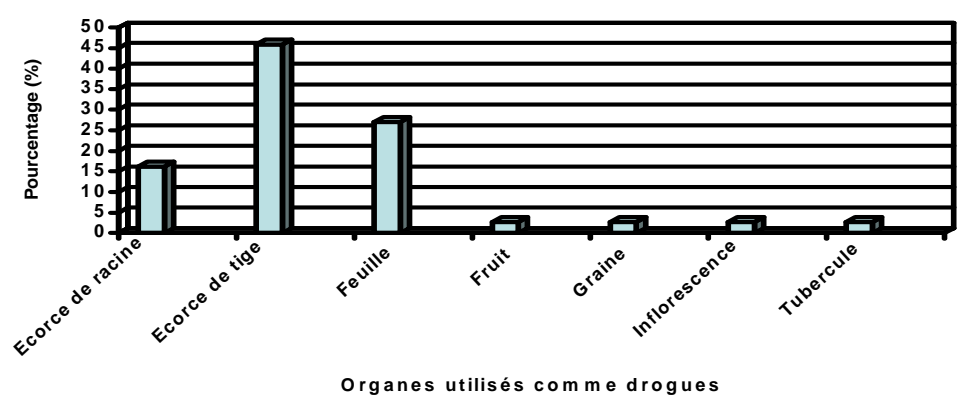

Figure 3 : Histogramme de la répartition des organes employés comme drogues.

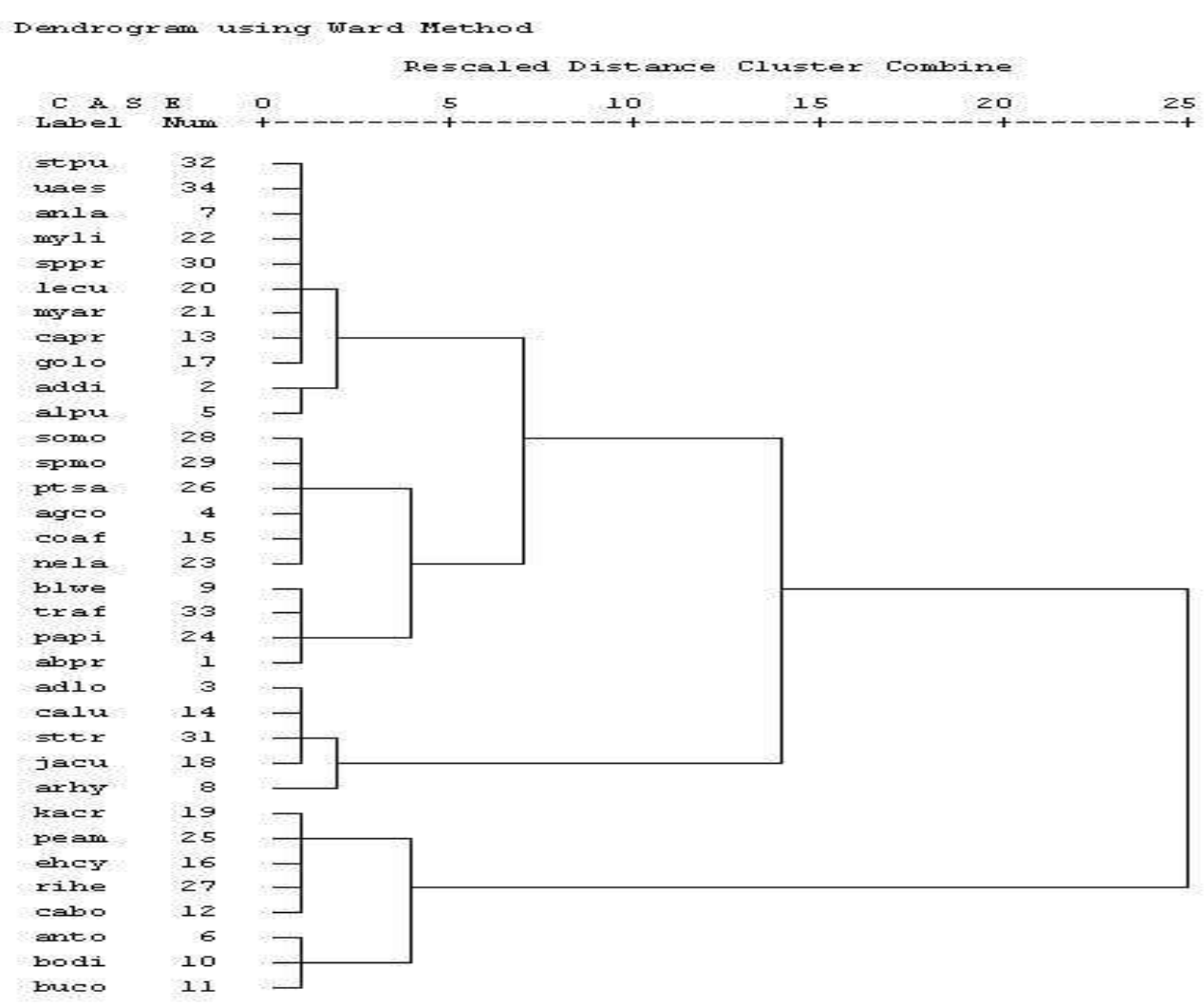

Figure 4 : Dendrogramme des indices d'efficacité des plantes servant à la préparation des remèdes. Les 4 lettres désignent les premières lettres du genre et de l'épithète spécifique de chaque espèce Les numéros afférents correspondent à l'ordre de citation des espèces, dans le tableau 1 
Tableau 1 : Monographie des plantes utilisées pour faciliter l'accouchement en pays Abbey et Krobou.

\begin{tabular}{|c|c|c|c|c|c|c|}
\hline Nom scientifique et famille & $\begin{array}{l}\text { Type } \\
\text { morphologique }\end{array}$ & Statut & $\begin{array}{l}\text { Organes } \\
\text { utilisés }\end{array}$ & Techniques de préparation & $\begin{array}{l}\text { Modes } \\
\text { d'administration }\end{array}$ & $\begin{array}{l}\text { Indices } \\
\text { d'efficacité }\end{array}$ \\
\hline Abrus precatorius (Fabaceae) & Liane & Rudérale & Feuille & Pilage avec kaolin : pâte + eau & Boisson & 70 \\
\hline Adansonia digitata (Bombacaceae) & Arbre & Sauvage & Ecorce de tige & Ramollissement, expression : jus & Boisson & 55 \\
\hline Adenia lobata $\quad$ (Passifloraceae) & Liane & Jachère & Racine & Macération aqueuse : macéré & Boisson & 47 \\
\hline Ageratum conyzoides (Asteraceae) & Herbe & Pluviale & Feuille & Pilage avec kaolin : pâte + eau & Boisson & 61 \\
\hline Alternanthera pungens (Amaranthaceae) & Herbe & Rudérale & Feuille & Pétrissage : pâte + eau & Purge & 53 \\
\hline Antiaris toxicaria (Moraceae) & Arbre & Sauvage & Ecorce de tige & Ramollissement, expression : jus & Boisson & 82 \\
\hline${ }^{{ }^{1} 1}$ Antidesma laciniatum (Euphorbiaceae) & Arbuste & Sauvage & Ecorce de tige & Pétrissage : pâte + eau & Boisson, purge & 58 \\
\hline Arachis hypogaea (Fabaceae) & Herbe & Cultivé & Feuille + tige & Pilage avec kaolin : pâte + eau & Boisson & 39 \\
\hline${ }^{* 2}$ Blighia welwitschii (Sapindaceae) & Arbre & Sauvage & Ecorce de tige & Pétrissage : pâte + eau & Purge & 66 \\
\hline Boerhavia diffusa (Nyctaginaceae) & Herbe & Rudérale & Tubercule & Pétrissage, expression : jus & Purge & 85 \\
\hline Buchholzia coriacea (Capparaceae) & Arbuste & Sauvage & Graine & Pétrissage : pâte + eau & Purge & 79 \\
\hline Caesalpinia bonduc (Caesalpiniaceae) & Liane & Sauvage & Feuille & Ramollissement, expression : jus & $\begin{array}{l}\text { Instillations } \\
\text { buccales }\end{array}$ & 73 \\
\hline${ }^{{ }^{* 1} \text { Carapa procera }(\text { Meliaceae })}$ & Arbuste & Sauvage & Ecorce de tige & Pétrissage : pâte + eau & Boisson, purge & 58 \\
\hline Carpolobia lutea (Polygalaceae) & Arbuste & Sauvage & Ecorce de racine & Décoction : décocté & Boisson & 47 \\
\hline Costus afer (Zingiberaceae) & Herbe & Jachère & Inflorescence & Pilage avec kaolin et 2 œufs : pâte + eau & Boisson & 61 \\
\hline${ }^{* 3}$ Ehretia cymosa (Boraginaceae) & Arbre & Forêt secondaire & Ecorce de racine & Pétrissage : pâte + eau & Purge & 74 \\
\hline${ }^{* 1}$ Gouania longipetala (Rhamnaceae) & Liane & Sauvage & Ecorce de tige & Pétrissage : pâte + eau & Boisson, purge & 58 \\
\hline Jatropha curcas (Euphorbiaceae) & Arbuste & Cultivée & Feuille & Ramollissement, expression : jus & $\begin{array}{l}\text { Instillations } \\
\text { buccales }\end{array}$ & 45 \\
\hline Kalanchoe crenata (Crassulaceae) & Herbe & Cultivée & Tige & Pétrissage avec muscade : pâte + eau & Purge & 76 \\
\hline${ }^{* 1}$ Lecaniodiscus cupanioides (Sapindaceae) & Arbuste & Sauvage & Ecorce de tige & Pétrissage : pâte + eau & Boisson, purge & 58 \\
\hline${ }^{* 1}$ Myrianthus arboreus (Cecropiaceae) & Arbre & Forêt secondaire & Ecorce de tige & Pétrissage : pâte + eau & Boisson, purge & 58 \\
\hline${ }^{* 1}$ Myrianthus libericus (Cecropiaceae) & Arbre & Forêt secondaire & Ecorce de tige & Pétrissage : pâte + eau & Boisson, purge & 58 \\
\hline Newbouldia laevis (Bignoniaceae) & Arbre & Forêt secondaire & Feuille & Pétrissage avec maniguette : pâte + eau & Purge & 60 \\
\hline
\end{tabular}


K. N'GUESSAN et al. / Int. J. Biol. Chem. Sci. 4(4): 1004-1016, 2010

\begin{tabular}{|c|c|c|c|c|c|c|}
\hline Paullinia pinnata (Sapindaceae) & Liane & Forêt secondaire & Fruit + racine & Pétrissage avec muscade : pâte + eau & Boisson & 67 \\
\hline Persea americana (Lauraceae) & Arbre & Cultivée & $\begin{array}{l}\text { Feuille + écorce } \\
\text { de tige }\end{array}$ & Ramollissement, expression : jus & $\begin{array}{l}\text { Instillations } \\
\text { buccales }\end{array}$ & 75 \\
\hline Pterocarpus santalinoides (Fabaceae) & Arbre & Sauvage & Ecorce de tige & Pétrissage : pâte + eau & Purge & 63 \\
\hline${ }^{* 3}$ Ricinodendron heudelotii (Euphorbiaceae) & Arbre & Sauvage & Ecorce de tige & Pétrissage : pâte + eau & Purge & 74 \\
\hline$*^{4}$ Solenostemon monostachyus (Lamiaceae) & Herbe & Rudérale & Feuille & Ramollissement, expression : jus & $\begin{array}{l}\text { Instillations } \\
\text { buccales }\end{array}$ & 62 \\
\hline$*^{4}$ Spondias mombin (Anacardiaceae) & Arbre & Forêt secondaire & Feuille & Ramollissement, expression : jus & $\begin{array}{l}\text { Instillations } \\
\text { buccales }\end{array}$ & 62 \\
\hline${ }^{* 1}$ Spondianthus preussii (Euphorbiaceae) & Arbre & Sauvage & Ecorce de tige & Pétrissage : pâte + eau & Boisson, purge & 58 \\
\hline Sterculia tragacantha (Sterculiaceae) & Arbre & Forêt secondaire & Ecorce de racine & Macération dans vin de palme & Boisson & 49 \\
\hline${ }^{* 1}$ Strombosia pustulata var. lucida (Olacaceae) & Arbre & Sauvage & Ecorce de tige & Pétrissage : pâte + eau & Boisson, purge & 58 \\
\hline$*^{2}$ Treculia africana var. africana (Moraceae) & Arbre & Sauvage & Ecorce de tige & Pétrissage : pâte + eau & Purge & 66 \\
\hline${ }^{* 1}$ Uapaca esculenta (Euphorbiaceae) & Arbre & Sauvage & Ecorce de tige & Pétrissage : pâte + eau & Boisson, purge & 58 \\
\hline
\end{tabular}

${ }^{*}$ : Recette plurispécifique faisant intervenir, en association, les 9 plantes suivantes : Antidesma laciniatum, Carapa procera, Gouania longipetala, Lecaniodiscus cupanioides, Myrianthus arboreus,

Myrianthus libericus, Spondianthus preussii, Strombosia pustulata et Uapaca esculenta

$*^{2}$ : Recette bispécifique faisant intervenir, en association : Blighia welwitschii et Treculia africana var. africana

$*^{3}$ : Recette bispécifique faisant intervenir, en association : Ehretia cymosa et Ricinodendron heudelotii

${ }^{*}{ }^{4}$ : Recette bispécifique faisant intervenir, en association : Solenostemon monostachyus et Spondias mombin 


\section{DISCUSSION}

\section{Caractéristiques botaniques}

La prédominance des 2 familles (Euphorbiaceae et Fabaceae) qui comptent le plus grand nombre de plantes médicinales, traditionnellement utilisées pour leurs effets ocytociques, s'expliquerait par le fait que ces familles font partie, du point de vue du nombre d'espèces qu'elles comportent, des plus importantes de la flore ivoirienne (AkéAssi, 2001). Les plantes recensées (34) représentent $9,41 \%$ des plantes répertoriées lors de l'étude ethnopharmacologique que nous avons effectué à Agboville (N'guessan, 2008). La comparaison de nos résultats avec d'autres travaux montre que les inventaires présentent les mêmes caractéristiques dans l'ensemble des études. Ce sont les Spermaphytes qui constituent l'essentiel de l'arsenal des plantes à effet ocytocique. Cependant, on observe une variabilité dans le nombre des individus recensés d'une étude à l'autre. On note chez Ouattara (2006), 03 plantes représentant $1,78 \%$ du répertoire des plantes recensées au cours de l'étude ethnopharmacologique menée en pays Dida, dans la région de Divo (Sud de la Côted'Ivoire). Cette représentativité n'a pas été observée au cours des enquêtes ethnobotaniques réalisées dans les forêts classées du Haut-Sassandra et de SCIO en Côte-d'Ivoire (Tra Bi, 1997). Dans son étude, l'auteur montre que 08 espèces de plantes $(8,88 \%)$ constituent l'essentiel de l'arsenal des taxons utilisés pour faciliter les accouchements. Zirihi (1991) a rapporté que les Bété du Département d'Issia (Centre-Ouest de la Côte-d'Ivoire) emploient 05 plantes, soit 4,31\% des plantes recensées. Vangah (1986) indique que les ethnies Akans de la région littorale de la Côte-d'Ivoire se servent de 24 espèces de plantes exerçant des effets ocytociques; ces plantes représentent 7,97\% des plantes recensées. Adjanohoun et AkéAssi (1979) font état de 5 espèces de plantes, soit $1,65 \%$ du répertoire des taxons recensés, lors de leur étude effectuée sur les plantes médicinales de Côte-d'Ivoire. Cette variabilité serait due aux variations dans les méthodes d'investigation ; elle pourrait notamment s'expliquer par les différences de localités et de végétation.

\section{Caractéristiques ethnopharmacologiques}

Les tiges et leurs écorces sont majoritairement utilisées $(45,92 \%)$, comme drogues. Ce résultat ne concorde pas avec celui de Tra Bi (1997) qui indique que les populations des forêts classées du HautSassandra et de SCIO, utilisent majoritairement les feuilles (70\%). Les techniques de prélèvement comme le déracinement, l'ébranchage et l'écorçage, sont des modes de récolte signalés par Ouattara (2006), dans son étude sur les plantes médicinales à Divo (Sud forestier de la Côted'Ivoire). L'auteur indique que l'écorçage laisse le plus souvent des cicatrices énormes par lesquelles ces plantes sont ultérieurement attaquées par les champignons, les oiseaux, les chenilles infestantes. Le déracinement, l'ébranchage et l'écorçage s'avèrent donc nocifs car ces modes de prélèvement empêchent la plante de parvenir à la floraison, induisent des infections et sont à l'origine de la menace de disparition des espèces (Anoma et Aké-Assi, 1989).

Les recettes médicamenteuses sont préparées à partir de drogues végétales, animales et minérales, ce qui montre que, pour soulager ses maux et blessures, l'homme a eu recours à son environnement immédiat, en accord avec les déclarations de Fleurentin (2007). On note 23 recettes médicamenteuses dont 19 , soit $82,60 \%$, sont monospécifiques. Cette prépondérance des recettes monospécifiques est à l'avantage des patients. Les associations de plantes, mal assorties, sont parfois dangereuses. En Afrique, environ 30\% des accidents mortels sont dus à l'usage des mixtures (N'guessan et al., 2009b). Le mode d'administration le plus sollicité est la boisson $(45,23 \%)$. Ce résultat diffère de celui que rapporte Ouattara (2006) au sujet des Dida de Divo (Côte-d'Ivoire) ; ces derniers emploient majoritairement les purges $(66,66 \%)$. Cette différence constatée au niveau des modes d'administration serait due aux différences de localités liées aux habitudes des peuples 
concernés, à leur tradition et à leurs préférences. On pense, en pays Abbey et Krobou que l'efficacité d'un remède peut être renforcée, grâce à des paroles magiques de nature incantatoire. Les incantations sont des jeux de mots qui constituent, avec les drogues préparées, le médicament. Par les incantations, on appelle la bénédiction sur le médicament. L'effet incantatoire, scientifiquement inexplicable, peut dépendre du guérisseur, selon qu'il a un esprit puissant ou non et des dieux invoqués. Toutes les médications ne font pas intervenir des incantations. Ci-après, nous évoquons un exemple d'incantation. «Sé biè prè nin djènin, naa a délè bo kroi » ce qui traduit, dans la langue locale Krobou, veut dire, «donnesnous l'enfant, pour qu'on sache que tu es puissant », lors du traitement, pour accouchement facile, faisant intervenir Ehretia cymosa et Ricinodendron heudelotii.

\section{Indices d'efficacité}

En fonction de leur indice d'efficacité, les plantes ont été soumises à une analyse hiérarchique, à travers un dendrogramme (figure 4). Cette analyse a permis d'établir 4 groupes de plantes, lorsque l'on réalise une coupe à la distance cluster de 5 . Le premier groupe est constitué des plantes ayant un indice d'efficacité compris entre 73 et 85 . Il s'agit des plantes suivantes: Buchholzia coriacea, Boerhavia diffusa, Antiaris toxicaria, Caesalpinia bonduc, Ricinodendron heudelotii, Ehretia cymosa, Persea americana et Kalanchoe crenata. Elles provoquent l'accouchement en moins de 30 minutes et possèdent le meilleur indice d'efficacité. Elles sont à conseiller, lors de la parturition. Le deuxième groupe a un indice d'efficacité compris entre 60 et 70 . Il s'agit des plantes suivantes: Abrus precatorius, Paullinia pinnata, Treculia africana var. africana, Blighia welwitschii, Newbouldia laevis, Costus afer, Ageratum conyzoides, Pterocarpus santalinoides, Spondias mombin et Solenostemon monostachyus. Elles provoquent l'accouchement, environ 40 minutes, après administration et viennent en deuxième position en ce qui concerne les indices d'efficacité. Dans le troisième groupe, on note les espèces suivantes: Alternanthera pungens, Adansonia digitata, Gouania longipetala, Carapa procera, Myrianthus arboreus, Lecaniodiscus cupanioides, Spondianthus preussii, Myrianthus libericus, Antidesma laciniatum, Uapaca esculenta et Strombosia pustulata var. lucida. Elles ont un indice d'efficacité variant de 53 à 58 et agissent assez tardivement, environ 50 minutes, après administration. Le quatrième et dernier groupe comporte les espèces suivantes: Adenia lobata, Carpolobia lutea, Sterculia tragacantha, Jatropha curcas et Arachis hypogaea. Elles ont un indice d'efficacité variant de 39 à 49 et agissent plus lentement, plus d'une heure après leur administration.

\section{Constat de recoupement et de divergence de résultats}

On note un constat de recoupement concernant quelques plantes exerçant des effets ocytociques. L'usage thérapeutique de certaines plantes de notre liste est reconnu chez d'autres groupes ethniques. Les écorces de tige de Gouania longipetala sont également utilisées pour faciliter l'accouchement chez les populations du Haut Sassandra et de Scio dans le Centre-Ouest de la Côte-d'Ivoire (Tra Bi, 1997). Dans les îles Maurices, on emploie aussi le décocté des écorces de tige de Persea americana, comme ocytocique (Anonyme, 1983). Les écorces de tige de Adansonia digitata sont employées par les habitants du Haut Plateau Central, au Burkina Faso, pour leur effet ocytocique (Nacoulma, 1996). L'utilisation des feuilles de Alternanthera pungens est signalée par Bouquet et Debray (1974).

A coté des similitudes, nous avons noté des divergences quant à l'usage de quelques plantes. Les Attié et les Oubi de Côte-d'Ivoire utilisent les feuilles de Abrus precatorius, pour empêcher l'avortement (Adjanohoun et Aké-Assi, 1979). Etant donné que les substances anti-abortives provoquent le silence utérin, l'effet induit est contraire à 
celui que nous indiquons dans la présente étude. Nous notons également une divergence quant à l'utilisation des feuilles de Spondias mombin. En effet, Kerharo et Adam (1974) signalent que les Manding du Sénégal oriental conseillent le décocté des feuilles de la plante aux femmes sujettes aux avortements répétés. Or, toute substance qui empêche un avortement inhibe les contractions utérines et ne peut donc être utilisée pour faciliter les accouchements. Nos résultats semblent en contradiction avec ceux des auteurs mentionnés plus haut. A Brazzaville (Congo), selon Binimbi-Massengo et al. (2007), Carpolobia lutea et Paullinia pinnata sont employées aussi contre les menaces d'avortement. Elles inhiberaient les contractions utérines, contrairement à l'usage signalé par les Abbey et les Krobou. Ces divergences montrent la nécessité d'étudier les effets d'extraits de ces plantes.

\section{Caractéristiques phytochimiques et pharmacologiques}

Nous avons procédé à une validation des pratiques médicales traditionnelles, en recherchant, pour quelques plantes, l'activité pharmacologique et la composition phytochimique qui permettraient d'expliquer leur effet ocytocique. Les feuilles de Abrus precatorius L. (Fabaceae) renferment des saponosides triterpéniques, des composés chimiques qui exercent des effets comparables à ceux des oestrogènes (Nacoulma, 1996), ce qui justifierait l'emploi de la plante en tant que ocytocique. On a isolé des écorces de tige de Adansonia digitata L. (Bombacaceae), un principe actif, l'adansonine, doué de propriété ocytocique (Nacoulma, 1996), ce qui est en accord avec l'usage traditionnel de la plante. Chez Ageratum conyzoides L. (Asteraceae) ou herbe aux sorciers, la facilitation de l'accouchement serait le fait des flavonoïdes (flavanones) qui exercent un effet ocytocique (Frantisek et al., 1973). Alternanthera pungens est riche en saponines triterpéniques, reconnues pour leur effet spasmogénique (Nacoulma, 1996). Selon Nacoulma (1996), les feuilles de Arachis hypogaea $\mathrm{L}$.
(Fabaceae) contiennent de la vitamine $\mathrm{B}_{1}$, responsable des spasmes musculaires notamment des muscles utérins; dès lors son efficacité dans la facilitation des accouchements est compréhensible. Sur le plan pharmacologique, Kerharo et Adam (1974) montrent que des extraits d'écorces de tige et de racine de Antiaris toxicaria Lesch. var. africana (Engl.) C.C. Berg. (Moraceae) produisent une contraction tonique marquée sur l'utérus isolé de lapine, ce qui concorde avec l'usage thérapeutique de la plante. Traditionnellement, les tubercules racinaires de Boerhavia diffusa sont recommandées aux parturientes, pour leur effet ocytocique. Les tests phytochimiques réalisés sur la drogue indiquent la présence d'alcaloïdes indoliques, probablement de type agroclavine; ces alcaloïdes sont doués de propriétés spasmogéniques sur les muscles lisses, notamment le muscle utérin (Bouquet et Debray, 1974). Les feuilles de Jatropha curcas sont riches en saponosides triterpéniques qui exercent un effet spasmogénique (Bouquet et Debray, 1974). Selon Adjanohoun et Aké-Assi (1979), Kalanchoe crenata (Andr.) Haw. (Crassulaceae) renferme de l'acide ascorbique (vitamine $\mathrm{C}$ ) qui se révèle comme un fortifiant, un stimulant de l'effort physique ; les feuilles de la plante peuvent être conseillées à la parturiente. Kerharo et Adam (1974) signalent que Paullinia pinnata L. (Sapindaceae) contient un saponoside dont l'aglycone triterpénique (principe actif) provoque un effet abortif; or ce qui est abortif entraîne des contractions fortes de l'utérus et peut, par conséquent, être utilisé pour faciliter l'accouchement chez la parturiente. Sur le plan pharmacologique, on a recherché l'activité musculotrope d'extrait de Persea americana, in vitro, sur l'utérus isolé de souris gravides. L'extrait stimule d'une manière significative, l'utérus isolé de souris, à la dose de 16,66 mg/ml (Anonyme, 1989). Selon Bantsiele et al. (2007), les lambeaux d'utérus isolé de lapine, placés dans une solution physiologique normale, développent des contractions spontanées et rythmiques. En 
présence de doses croissantes d'extrait de Sterculia tragacantha $\left(10^{-5}, 10^{-4}\right.$ et $5.10^{-4}$ $\mathrm{g} / \mathrm{ml}$ ), il se manifeste un accroissement de l'amplitude des contractions rythmiques et leurs fréquences, ce qui s'accorde avec l'utilisation traditionnelle de la plante, en tant que ocytocique.

\section{Conclusion}

Les investigations ethnopharmacologiques menées dans le Département d'Agboville, en Côte-d'Ivoire, montrent que 34 espèces de plantes sont employées par les Abbey et Krobou, pour faciliter l'accouchement. Diverses parties de la plante servent à mettre au point des préparations médicamenteuses, en général monospécifiques. La préparation à l'accouchement facile relève de la compétence des naturothérapeutes. Des paroles incantatoires viennent parfois s'ajouter aux remèdes mis au point à partir des ressources naturelles. Les effets induits par les plantes, pour faciliter l'accouchement, sont le fait de divers groupes chimiques: acide ascorbique, adansonine, alcaloïdes indoliques (agroclavine), flavonoïdes (flavanones), saponosides triterpéniques et vitamine $\mathrm{B}_{1}$, qui constituent la base scientifique de l'utilisation thérapeutique traditionnelle des plantes étudiées.

\section{REFERENCES}

Adjanohoun EJ, Aké-Assi L. 1979. Contribution au recensement des plantes médicinales de Côte-d'Ivoire. Université d'Abidjan, Centre National de Floristique, $358 \mathrm{p}$.

Aké-Assi L. 2001. Flore de la Côte-d'Ivoire: catalogue systématique, biogéographique et écologique. Boissiera, Conservatoire et Jardin Botanique de Genève, 396 p.

Anoma G, Aké-Assi L. 1989. Flore de la Côte-d'Ivoire : disparition de nombreuses espèces due à la destruction inconsidérée de l'espace naturel: le cas de Monanthotaxis capea (E. G. et A. Camus) Verdc. (Annonaceae). Bulletin de liaison de 1'Agence de Coopération Culturelle et
Technique. Méd. Trad. Pharm., 3(2): 153-158.

Anonyme. 1983. Médecine Traditionnelle et Pharmacopée, Contribution aux Études Ethnobotaniques et Floristiques à Maurice (Îles Maurice et Rodrigue). Edition de l'Agence de Coopération Culturelle et Technique ; 82-83.

Anonyme. 1989. Vers une Pharmacopée Caraïbe. Edition de l'Agence de Coopération Culturelle et Technique; 274- 277.

Anonyme. 1994. Enquête démographique et de santé de 1991 à 1993. Institut National de la Statistique. Association Ivoirienne pour le Bien-être de la Famille, Treichville (Côte-d'Ivoire), pp 108-109.

Anonyme 1999. Plan d'aménagement de la forêt classée de Bamo (Agboville). Société de Développement des Plantations Forestières, p. 1-22.

Bantsiélé GB, Tchicaya FO, Mbemba C, Datté YJ, Offoumou AM. 2007. Effet pharmacologique de l'extrait aqueux de Sterculia tragacantha (Sterculiaceae) sur l'activité contractile de l'intestin isolé de lapin et de l'utérus isolé de souris gestante. Revue Méd. Pharm. Afr., 20: 125-132.

Binimbi-Massengo A, N'Zongi J, Abena AA, Diatewa M. 2007. Inventaire des plantes médicinales utilisées dans le traitement des menaces d'avortement à Brazzaville. Revue Méd. Pharm. Afr., 20: 155-164.

Bouquet A, Debray M. 1974. Plantes Médicinales de Côte-d'Ivoire. Imprimerie Louis Jean : Paris (France) ; 13-88.

Fleurentin J, Hayon JC, Pelt JM. 2007. Les Plantes qui nous Soignent - Traditions et Thérapeutique. Editions Ouest-France ; 7-14.

Folliard T. 2008. Phytothérapie externe en Amérique du Sud et en Amérique Centrale-Mexique et Guatemala. Revue Méd. Pharm. Afr., 20: 175-183.

Frantisek S, Vaclav J. 1976. Plantes Médicinales: Atlas illustré. Edition Artia : Prague ; 247 p. 
Kamanzi A. 2002. Plantes médicinales de Côte-d'Ivoire : investigations phytochimiques guidées par des essais biologiques. Thèse de Doctorat d'Etat, Université de Cocody-Abidjan, 176 p.

Kerharo J, Adam JG. 1974. La pharmacopée Sénégalaise Traditionnelle. Plantes Médicinales et Toxiques. Edition Vigot Frères : Paris ; 143-589.

Koné MW. 2005. Potentiel des plantes médicinales de Côte-d'Ivoire dans le contrôle des haemonchoses chez les ovins. Thèse de Doctorat de l'Université de Cocody, Abidjan, 202 p.

Nacoulma-Ouédraogo O. 1996. Plantes médicinales te pratiques médicales traditionnelles au Burkina Faso : cas du Plateau central. Thèse de Doctorat ès Sciences Naturelles, Université de Ouagadougou, Burkina-Faso, p 26-54.

N'Guessan K. 2000. La lutte contre la tragédie de l'accouchement par les plantes. Bioterre, Rev. Inter. Sci. de la Vie et de la Terre, 1(1): 57-66.

N'Guessan K. 2008. Plantes médicinales et pratiques médicales traditionnelles chez les peuples Abbey et Krobou du Département d'Agboville (Côte-d'Ivoire). Thèse de Doctorat ès Sciences Naturelles. Université de Cocody-Abidjan, U.F.R. Biosciences, Laboratoire de Botanique. $\mathrm{N}^{\circ}$ d'ordre: $561 / 2008,235 \mathrm{p}$.

N'guessan K, Kouassi KE, Tiebré MS. 2009a. Plantes utilisées dans le traitement des troubles gynéco-obstétriques par les peuples Abbey et Krobou d'Agboville (Côte-d'Ivoire). Phytothérapie, 7: 262274.

N'Guessan K, Tra BFH, Koné MW. 2009b. Etude ethnopharmacologique de plantes antipaludiques utilisées en médecine traditionnelle, chez les Abbey et Krobou d'Agboville (Côte-d'Ivoire). Ethnopharmacologia, 44: 42-50.
Ouattara D. 2006. Contribution à l'inventaire des plantes médicinales significatives utilisées dans la région de Divo (sud forestier de la Côte-d'Ivoire) et à la diagnose du poivrier de Guinée : Xylopia aethiopica (Dunal) A. Rich. (Annonaceae). Thèse de Doctorat Université de Cocody-Abidjan (Côted'Ivoire), $184 \mathrm{p}$.

Sournia G, Arnaud JC. 1978. Les Ethnies de Côte-d'Ivoire. In Atlas de la Côted'Ivoire. Edition Groupe J.A.-51 Avenue des Ternes-75017 : Paris ; 26-27.

Tra Bi FH. 1997 Utilisation des plantes, par l'homme, dans les forêts classées du Haut-Sassandra et de Scio, en Côted'Ivoire. Thèse de Doctorat de $3{ }^{\text {ème }}$ Cycle, Université de Cocody-Abidjan, 212 p.

Vangah-Manda MO. 1986. Contribution à la connaissance des plantes médicinales utilisées par les ethnies Akans de la région littorale de la Côte-d'Ivoire. Thèse de Doctorat de $3^{\text {ème }}$ Cycle, Université Nationale de Côte-d'Ivoire, d'Abidjan, $464 \mathrm{p}$.

Zirihi GN. 1991. Contribution au recensement, à l'identification et à la connaissance de quelques espèces végétales utilisées dans la médecine traditionnelle et la pharmacopée chez les Bété du Département d'Issia, Côted'Ivoire. Thèse de Doctorat de $3^{\text {ème }}$ Cycle, Université d'Abidjan, 150 p.

Zirihi GN. 2006. Études botanique, pharmacologique et phytochimique de quelques plantes médicinales antipaludiques et/ou immunogènes utilisées chez les du Département d'Issia, dans l'Ouest de la Côte-d'Ivoire. Thèse de Doctorat d'Etat, Université de CocodyAbidjan, 126p. 